\title{
A Culture of Quality in Higher Education
}

\author{
Jorge Ortiz-Herrera ${ }^{1}$; Susana Cadena-Vela ${ }^{1}$; Tania Gualli ${ }^{2}$ Irma García-Serrano ${ }^{1}$; Rodrigo Padilla-Verdugo ${ }^{3}$; \\ ${ }^{1}$ Universidad Central del Ecuador, Ecuador, jdortiz@uce.edu.ec, scadena@uce.edu.ec, iggarcia@uce.edu.ec, \\ ${ }^{2}$ Escuela Politécnica Nacional, Ecuador, tania.gualli@epn.edu.ec, \\ 33niversidad de Cuenca, Ecuador, rodrigo.padilla@ucuenca.edu.ec
}

\begin{abstract}
In Ecuador the discussion on the quality of higher education has been put on the stage in the last fifteen years, although the initial motivation is present in the times of application of the processes of evaluation with the purpose of accreditation of the universities, these events have been carried out in a mandatory way in three occasions. The universities try to obtain the best results and with it the institutional accreditation; however, in the intermediate times the efforts are dissipated or forgotten. For this reason, universities must reorient their teaching, research, networking and management activities so that they are carried out with quality on a permanent basis. The responsibility for good management is fundamentally in the hands of the authorities; however, the participation of the entire educational community is also necessary in order to promote what is called "quality culture". For the concretion of this culture of quality, a system of quality management is required as a tool that offers the possibility of evaluating oneself, having timely and precise information for university planning and action. The system itself does not constitute the exercise of quality for higher education, but is the tool that allows actors to focus with quality in the practice of their daily functions and thus create a quality culture that allows the university to achieve high standards of compliance in their substantive functions. A means for this is the validation of a system of indicators in various dimensions as a valid, living and dynamic proposal, which demands the participation of all sectors involved while giving them feedback, in this context this research work is done with a theoretical review that served to create a set of indicators, to proceed with its validation through discussion groups, a qualitative analysis of the opinions of the institutions involved in the study is presented, the results are presented the aspects to be considered within a system of quality management in Higher Education, in order to create an environment of quality culture.
\end{abstract}

Keywords- Quality culture, Quality system, Indicators, Quality of higher education.

Digital Object Identifier (DOI):

http://dx.doi.org/10.18687/LACCEI2020.1.1.364

ISBN: 978-958-52071-4-1 ISSN: 2414-6390

$1^{\text {th }}$ LACCEI International Multi-Conference for Engineering, Education, and Technology: "Engineering, Integration, and Alliances for a Sustainable Development" "Hemispheric Cooperation for Competitiveness and Prosperity on a Knowledge-Based Economy”, July 27-31, 2020, Virtual Edition. 


\title{
Cultura de Calidad en la Educación Superior
}

\author{
Jorge Ortiz-Herrera $^{1}$; Susana Cadena-Vela ${ }^{1}$; Tania Gualli²; Irma García-Serrano ${ }^{1}$; Rodrigo Padilla-Verdugo ${ }^{3}$; \\ ${ }^{1}$ Universidad Central del Ecuador, Ecuador,jdortiz@uce.edu.ec, scadena@uce.edu.ec,iggarcia@uce.edu.ec, ${ }^{2}$ Escuela \\ Politécnica Nacional, Ecuador, tania.gualli@epn.edu.ec, ${ }^{3}$ Universidad de Cuenca, Ecuador, rodrigo.padilla@ucuenca.edu.ec,
}

\begin{abstract}
Resumen. - En Ecuador la discusión sobre la calidad de la educación superior se ha puesto en escena en los últimos quince años debido a la aplicación de los procesos de acreditación de las universidades. En este marco, las universidades tratan de obtener los mejores resultados y con ello lograr la acreditación institucional; sin embargo, en los tiempos intermedios los esfuerzos se disipan o se olvidan. La responsabilidad de una buena gestión está fundamentalmente en manos de las autoridades; sin embargo, es necesaria también la participación de toda la comunidad educativa para promover lo que se denomina "cultura de calidad". Un medio para esto es la validación de un sistema de indicadores que sirvan como referencia para el trabajo continuo, por tanto, se plantea un conjunto de indicadores organizado en varias dimensiones como una propuesta valida, viva y dinámica, que demanda la participación de todos los sectores involucrados a la vez que los retroalimenta, en favor de fortalecer la cultura de calidad en las universidades. El presente trabajo de investigación se desarrolla utilizando la metodologíaí interpretativa (hermenéutica) que parte de una revisión teórica para crear un conjunto de indicadores que fueron validados mediante grupos de discusión bajo principios del enfoque cualitativo. En nuestros resultados se presentan aspectos a considerar dentro de un sistema de gestión de la calidad en la Educación Superior, en pro de crear un ambiente de cultura de calidad.
\end{abstract}

Abstract. - In Ecuador, the discussion on the quality of higher education has come to the fore in the last fifteen years due to the implementation of university accreditation processes. In this framework, universities try to obtain the best results and thus achieve institutional accreditation; however, in the intervening times efforts are dissipated or forgotten. The responsibility for good management is fundamentally in the hands of the authorities; however, the participation of the entire educational community is also necessary to promote what is called "quality culture". One way to do this is to validate a system of indicators that serve as a reference for ongoing work. Therefore, a set of indicators organized in various dimensions is proposed as a valid, living, and dynamic proposal that demands the participation of all sectors involved while providing them with feedback in order to strengthen the culture of quality in universities. This research work is developed using the interpretative methodology (hermeneutics) that starts from a theoretical review to create a set of indicators that were validated through discussion groups under the principles of the qualitative approach. Our results present aspects to be considered within a system of quality management in Higher Education, in order to create an environment of quality culture

Palabras clave. - Cultura de calidad, Sistema de calidad, Indicadores, Calidad de la educación superior

Keywords. - Quality culture, Quality system, Indicators, Quality of higher education

Digital Object Identifier (DOI):

http://dx.doi.org/10.18687/LACCEI2020.1.1.364

ISBN: 978-958-52071-4-1 ISSN: 2414-6390

\section{INTRODUCCIÓN}

La educación superior es una responsabilidad que no se limita a las Universidades, sino que también intervienen varios, organismos oficiales, redes académicas, y el mismo Estado, como actores corresponsables de su implementación y de llevar adelante actividades de docencia, investigación y vinculación con la sociedad [1]. Las interrogantes, para el sistema de educación superior, surgen cuando se debe responder si estas actividades se realizan de forma, eficiente, eficaz y de manera oportuna. ¿Qué significa "hacer bien" la tarea universitaria, o cómo determinar que ésta se ha realizado de forma correcta y eficiente?

En el Ecuador por varios años se ha creído que toda actividad universitaria, por el hecho de implementarse en las universidades [1], se las realizaba con calidad. Sin embargo, los procesos de evaluación implementados en los últimos doce años han puesto en evidencia que aquella afirmación no era del todo cierta. Tal es así que, algunas universidades fueron cerradas por mala calidad, mientras que a otras se les exigió, a veces recurrentemente, la generación de planes de mejora [2].

Los modelos que se utilizaron para evaluar la calidad de la educación superior estuvieron dirigidos a la acreditación [3] y las universidades reaccionaron en función de este objetivo. Así, las Instituciones de Educación Superior (IES) observaron el modelo y los indicadores a los que debían someterse, prepararon la información y evidencias en el sentido de obtener el mejor resultado; y una vez alcanzada la meta, la presión bajó y se regresó a las mismas prácticas comunes hasta que llegue el nuevo período de acreditación.

Sin embargo, desde varios sectores de la academia se han generado espacios de reflexión dirigidos a tratar a la calidad de la educación superior como una necesidad [4], como una actividad permanente, con responsabilidad compartida entre los diferentes actores universitarios. Esta responsabilidad no puede quedar reducida a la circunscripción de cumplir con el Estado, sino que se debe también a otros ámbitos como el sector privado, que en definitiva luego aprovecha al talento humano formado en las universidades; $y$ en definitiva constituye una corresponsabilidad social, puesto que es una de las formas, tal vez la única, que asegura el progreso de la sociedad. Así, la sociedad confía a las universidades la formación de los profesionales, pero debería también exigir que la academia partícipe en la solución de la problemática cotidiana mediante actividades de investigación y vinculación con la sociedad.

El Estado por su parte debe permitir un escenario con un marco regulatorio que permitan que las IES y los organismos conexos interactúen en correspondencia con las funciones sustantivas a ellas obligadas y que, conforme al entorno 
específico de esta misión, la gobernanza interna de cada IES genere los resultados esperados. Cuando se trata de los actores internos, es necesario analizar los elementos que permitan crear una Cultura de Calidad en el sistema. Una que oriente a autoridades, docentes, estudiantes, empleados y trabajadores hacia una participación permanente, consciente, y comprometida en la búsqueda y práctica de la calidad de la educación superior [5] .

La implementación de un Sistema de gestión de calidad en las IES permitirá la planificación, organización, ejecución y evaluación permanente de calidad [6]. Un sistema de esta naturaleza debe ser un elemento indispensable en manos de las autoridades universitarias. Para docentes, investigadores y estudiantes los indicadores de este sistema deben ser vectores para el desarrollo de sus actividades. El conocimiento del estado en cada indicador proporciona información de cumplimiento, y permitirá reconocer fortalezas y debilidades en la tarea universitaria.

La presente investigación se orienta a responder la pregunta $i \mathrm{La}$ existencia de indicadores construidos participativamente pueden contribuir a generar una cultura de calidad en la Educación superior?, para lo cual se trabajó con actores universitarios, poniendo a discusión un sistema de indicadores para gestionar la calidad, orientar el establecimiento de elementos claves para un proceso de mejora continua en la academia y a su vez generar una cultura de calidad que valore su historia, que sea dinámica y permanente.

\section{TRABAJOS RELACIONADOS}

El concepto de calidad es algo complejo y requiere de un largo y gran recorrido para ser comprendido tanto en la vida económica como en la vida social de una organización [7]. Según la norma ISO 9001, la calidad es entendida como el grado en el que un "conjunto de características inherentes cumple con los requisitos." Otra aportación significativa de la Norma ISO 9001 es la aplicación de los conceptos PlanificarHacer-Verificar-Actuar, metodología que Deming [6] que propone un "círculo virtuoso de la mejora continua", cuyo objetivo es el logro de un aumento en la satisfacción del cliente o usuario mediante el cumplimiento de sus requisitos. [8].

En las últimas tres décadas, los discursos sobre la calidad se han fundamentado en una variedad de definiciones y la búsqueda de métodos de medición, apuntando a la mejora o a la optimización [9]. Esta discusión sobre la calidad se traslada también al subsistema "educación superior", en donde el reto consiste en conocer si las IES son veraces en cuanto a lo que es 'ser una institución de enseñanza superior', sus funciones, productos y resultados. Le corresponde así mismo identificar procesos de valoración de dicha calidad e identificar si la medición de sus distintas facetes corresponden a indicadores cualitativos o cuantitativos [10].

La calidad de la educación superior nace en la década de los noventa como un sinónimo de mejoría y superación, asociada a la eficiencia y eficacia frente a la tensión con las demandas crecientes de expansión de la cobertura universitaria.
Esta génesis estaría determinada por el comportamiento de algunos factores internos y externos (educación de los docentes, nuevos estudiantes, métodos de gestión administrativa, acceso al mercado laboral, etc.)[10]. Sin embargo, en este debate aún no se logran acuerdos ni bases comunes sobre los factores, dimensiones, indicadores y procesos que puedan recoger lo que es calidad de la educación superior; por lo cual, en la actualidad existen diversos enfoques y significados.

Se podría decir que el concepto de calidad aplicado a la educación superior se asocia directamente con el éxito que tengan los egresados en el mercado de trabajo. Es decir, los interesados en recibir una educación superior de calidad esperan que el conocimiento y la formación recibidos estén en concordancia con las habilidades y competencias demandadas en el mercado laboral y las necesidades regionales y globales [8].

Por otro lado, los protagonistas del debate: docentes, investigadores, autoridades universitarias, representantes gubernamentales y agrupaciones de estudiantes, han intentado definir algunos mecanismos de valoración como por ejemplo los procesos de evaluación y acreditación con atención a ciertos criterios y parámetros básicos. Asegurando así perfiles y dedicación de la planta docente, equipamiento y desarrollo de infraestructura para la gestión de los programas curriculares, entre otros.

En América Latina, se tienen modelos de acreditación centrados en lo administrativo y no en el desempeño; los procesos de acreditación tienden a ser una simulación; se incrementa el aparato burocrático con la acreditación; falta participación real de la comunidad educativa; falta mayor credibilidad en los organismos acreditadores; y falta una visión compartida en torno a la acreditación como proceso de innovación y de transformación de las personas y comunidades acorde con los retos de la sociedad del conocimiento [4].

Para el caso de Ecuador, el antiguo CEAACES (Consejo de Evaluación, Acreditación y Aseguramiento de la Calidad de la Educación Superior del Ecuador), en un intento de normalizar ciertos procesos, empezó a trabajar en la evaluación y categorización de las IES con el objetivo de certificar su calidad. Muchos de estos procesos produjeron una creciente diferenciación institucional entre las universidades que fueron acreditadas y las que no; provocando incluso cierta discriminación para los estudiantes cuando acceden a su vida profesional [11].

Así, el tema de calidad cruza por varios paradigmas, tales como [12]: alcanzar metas propuestas, cumplir un proceso normativo, satisfacer estándares internacionales, obtener certificaciones, promover una educación superior centrada en la tarea pedagógica, mejorar metodologías y prácticas de su personal docente, etc. Sea cual fuere la opción, la búsqueda de la calidad sigue en construcción y es un tema vigente.

Cada IES actualmente cuenta con un conjunto de referentes simbólicos, históricos y un lenguaje que les permite hablar sobre la gestión de su calidad, pero la cultura de calidad va más 
allá de cumplir con estándares. La cultura tiene estrecha relación con la gestión, con el contexto local, con las prácticas y comportamientos de cada uno de los actores. La existencia de "culturas de calidad " se configuran a partir de creencias, valores, representaciones y prácticas compartidas y socializadas [9]. La cultura de calidad se convierte así en un proceso a ser construido entre todos los estamentos de las IES.

Una cultura de calidad en la educación superior debería incluir "lo ético y lo político como parte indisoluble de la filosofía que inspira los planes de estudio universitarios con el fin de promover la formación de sujetos conscientes, situados y preparados para participar en la vida comunitaria desde los principios de justicia social, equidad y sostenibilidad" [13]. Es de suma importancia que se construya esta cultura como una forma permanente de vida [14].

Al respecto, es importante citar un estudio que permitió conocer la situación de 21 sistemas de gestión de calidad implementados en la Universidad Autónoma de Baja California (UABC), en México. En los resultados de la estadística descriptiva del estudio se detectó que, del total de la muestra, 17 sistemas estaban certificados bajo la Norma ISO 9001: 2008 y uno en proceso de serlo. Por otra parte, el hallazgo relevante fue la ausencia de procesos relacionados con las actividades académicas. Los datos obtenidos de la investigación aportaron evidencia cuantitativa y cualitativa para concluir, entre otros cosas, que "en la UABC no se promueve el desarrollo de una cultura de la calidad con la implementación de sistemas de gestión de la calidad mediante un liderazgo comprometido" [8].

Estos datos sugieren la necesidad de realizar trabajos de investigación relacionados con la gestión de la calidad en las IES en contexto nacionales. Esto permitirá obtener evidencia sobre si los sistemas de gestión de calidad implementados se utilizan como una herramienta valiosa para el seguimiento y mejoramiento de la calidad, o bien, si solo se utilizan para cumplir con el requisito de una política pública.

La importancia de un sistema de gestión de la calidad radica primordialmente en el mejoramiento continuo, ya que su ausencia no permite medir en dónde estamos y si las metas estratégicas están avanzando. Características adicionales del sistema radican en: el mejoramiento de procesos, involucramiento del personal en actividades diarias, mayor productividad y motivación [15], uso de herramientas de planeación y gestión para sistematizar procesos, incorporación de indicadores en la toma de decisiones [16]. Así el sistema se convierte en un catalizador que acelera el cambio de cultura de una organización [17].

La calidad y la cultura de la calidad, no es el fin de la educación superior, es solo el medio que motivará a cumplir la misión y visión institucional. Es una estrategia que dice que debes cumplir con tu responsabilidad primaria de hacer de mejor manera lo que planeaste con los recursos que han sido confiados [9].

Con estos antecedentes el estudio se orientó a recabar los aspectos comunes de los actores educativos de las universidades ecuatorianas acerca de la importancia en la implementación de un sistema de indicadores que permitan gestionar la calidad en las IES y favorecer el camino hacia una cultura de calidad.

\section{METODOLOGÍA}

El trabajo de investigación se desarrolló bajo un enfoque interpretativista, por la naturaleza de sus datos eminentemente cualitativos. Partiendo del interés del estudio por recabar directamente información de los actores universitarios, sus creencias, su visión del mundo y su cultura, la metodología escogida permite que estas concepciones no sean segmentadas. Al contrario, estos elementos tienen que ser comprendidos como una totalidad, considerando que la sola cuantificación de la realidad humana no permite entender cómo está funcionando el objeto de estudio. La investigación interpretativista pretende dar importancia al contexto, a la función y al significado de los actos humanos [18], valora la realidad de cómo es vivida y percibida una realidad, con las ideas, sentimientos y motivaciones de sus actores [19].

En este sentido, se utilizó un muestreo no probabilístico, el cual consiste en seleccionar aquellos casos accesibles y proximidad de los sujetos para el investigador, además de los siguientes aspectos:

a) Un cambio en la sensibilidad investigadora, ya que en el estudio se tomó en cuenta la realidad histórica del país, orientados por el objeto de la investigación, pero considerando el entorno cultural en donde se desarrolló el trabajo.

b) La investigación tuvo un componente teórico que incluye la tendencia internacional así como un análisis de las decisiones políticas emanadas por el Estado en temas de Educación Superior, incluyendo una revisión teórica [1].

c) Una participación de los actores universitarios con la representación de 14 universidades ecuatorianas.

Con estos elementos, se trabajó con 7 grupos de discusión, cada uno de ellos conformados por entre 5 y 7 expertos, con un formato de grupo focal para estimular el debate e intercambio de experiencias se obtenía la información en un ambiente agradable. La duración de cada grupo focal fue de 4 horas. La población interviniente en el proceso de investigación estuvo conformada por los responsables de las unidades de aseguramiento de la calidad, evaluación interna, coordinadores de procesos de planificación y acreditación, asesores, y autoridades de varias universidades del Ecuador, con un total de 45 participantes.

Las discusiones fueron grabadas y tuvieron tres preguntas directrices: ¿Un proyecto de fortalecimiento de la cultura de calidad es importante en la Educación Superior?, ¿Qué aspectos importantes debería incluirse en un proyecto de esta naturaleza? y ¿Qué aspectos son mejorables en la propuesta presentada?

Para el desarrollo de los grupos de discusión en relación con las cuestiones planteadas, se siguió las siguientes directrices:

- Se partió con la presentación de los conceptos de calidad en la educación superior y algunos indicadores que respondieron a una revisión teórica realizada [20]. 
- El grupo de investigadores presentó las cuestiones de debate en cada grupo, se receptaron todas las observaciones y sugerencias a partir de la discusión entre los expertos.

- Cada coordinador de grupo presentó en plenaria sus aportes y se generó una discusión colectiva.

- Se codificaron los aportes de cada uno de los grupos para proceder a fusionarlos y generar conclusiones.

El estudio preliminar [20] de la investigación proporcionó una estructura para un sistema de indicadores, el cual está organizado en 6 dimensiones: Docencia (16 indicadores), Estudiantes (23), Gestión (20), Investigación (19), Recursos e infraestructura (18) y Vinculación con la Sociedad (11). Cada dimensión a su vez agrupa varias subdimensiones según su naturaleza. En cada ejercicio de discusión y validación los indicadores fueron sometidos a ser ratificados, redefinidos, eliminados o incorporar nuevos, considerando que los indicadores son dinámicos.

Para la validación de la construcción de cada indicador se entregó un formato, denominado "Ficha metodológica del indicador", que incluye su denominación, codificación, tipo de indicador (cuantitativo o cualitativo), una breve definición del indicador, la forma de cálculo, la definición de las variables relacionadas, el fundamento teórico del indicador, el análisis propuesto (que en general tiene tres niveles de aceptación: resultados óptimo, aceptable y débil), la unidad de medida, fuente de datos o información, periodicidad, serie cronológica, nivel de desagregación, referencia bibliográfica, fecha de elaboración y fecha de última revisión.

En la validación de cada indicador y del sistema en general se solicitó en primer lugar definir el "nivel de posicionamiento" que tiene cada indicador, las alternativas fueron: estratégico, táctico y operativo. Estratégico si el indicador se orienta a cumplir con los objetivos estratégicos de la IES [21]. Táctico si se orientan a cumplir objetivos puntuales y de resultados parciales. Operativo cuando el indicador refleja el cumplimiento de tareas periódicas y repetitivas pero que, a su vez, varios de ellos, aportan a los objetivos tácticos.

En los talleres también se trabajó sobre una definición del denominado "nivel de importancia", en función de la atención que debe dar cada IES a su valoración, los niveles propuestos son: esencial, significativo, optativo y no aplicable. El indicador es esencial cuando sostiene a las actividades sustantivas de la academia, es decir las que tienen una directa responsabilidad en la concreción de la docencia, investigación y vinculación. Es significativo cuando su aporte a las actividades sirve para consolidarlas, fortalecerlas y consolidan el proyecto universitario. Se consideran indicadores optativos a los que serán asumidos por la universidad para desarrollar planes o programas de ámbito particular, que sin ser de cumplimiento general del sistema una IES asume ante sí. Existe la posibilidad de que algún indicador no aplique a una IES, puesto que en su autodeterminación no tiene como objetivo trabajar en dicho ámbito, sin que esto afecte al cumplimiento de las actividades sustantivas, o por otro lado si el indicador se encuentra fuera de enfoque del sistema de gestión de calidad, en el último caso o se redefine o se elimina el indicador.

Los talleres buscaron que se realice el análisis en especial de la definición, forma de cálculo, fundamento y análisis del indicador previa una valoración con la escala de Likert (de 1, apreciación negativa a 5, apreciación positiva), con ello se obtuvo aportes de valía para la mejora de la propuesta del sistema de gestión de calidad.

La presentación en plenaria de las discusiones de cada grupo permitió lograr un lenguaje común en los aportes de los diferentes participantes e incluso en la aclaración de conceptos que la propuesta establece para su futura aplicación, considerando la importancia de establecer una cultura de calidad en la IES.

\section{RESULTADOS}

Los aspectos más importantes que fueron presentados en la plenaria se describen a continuación:

A) Valía e Importancia de un proyecto de Indicadores para el Sistema de Gestión de la Calidad en las IES

En la Figura 1, se encuentra determinados los cinco aspectos más importantes en relación con la valía e importancia del Sistema de Gestión de la Calidad presentado.

El primer aspecto y el más importante que resalta en la validación del catálogo de indicadores considerado por los participantes es la Mejora continua de la calidad (puntaje 10), la misma es considerada un aspecto clave para la mejora de la calidad en la gestión de educación superior porque presenta una guía en la aplicación de criterios de mejora y efectividad dentro de la gestión universitaria.

El segundo aspecto es la Propuesta participativa (puntaje 8), la cual recoge visiones reales, lo que involucra considerar indicadores válidos y aceptables dentro de las normas de gestión y evaluación de las Instituciones de Educación Superior. 


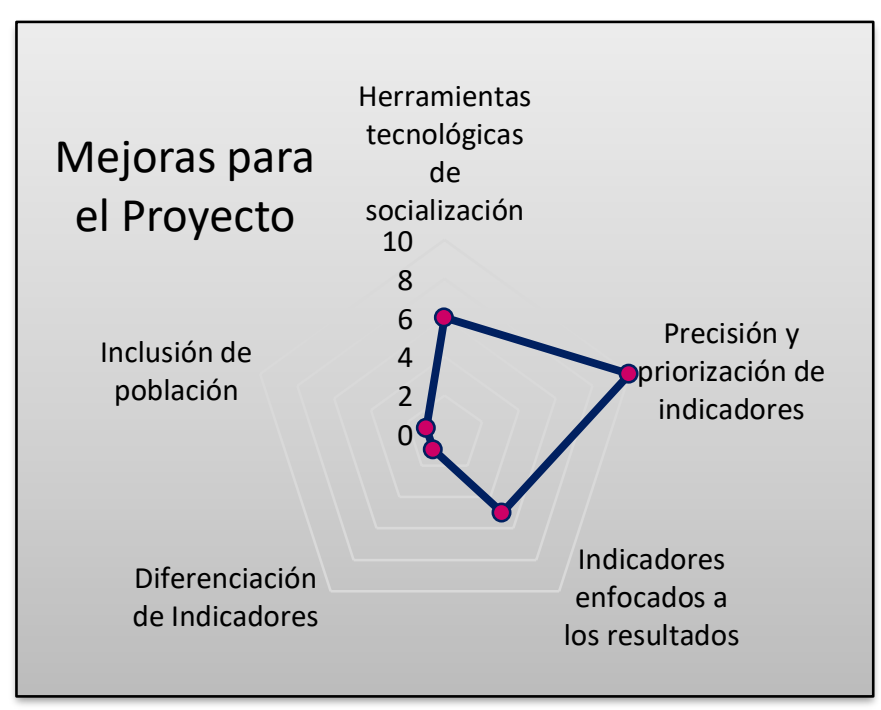

Figura 1. Valía e importancia del proyecto

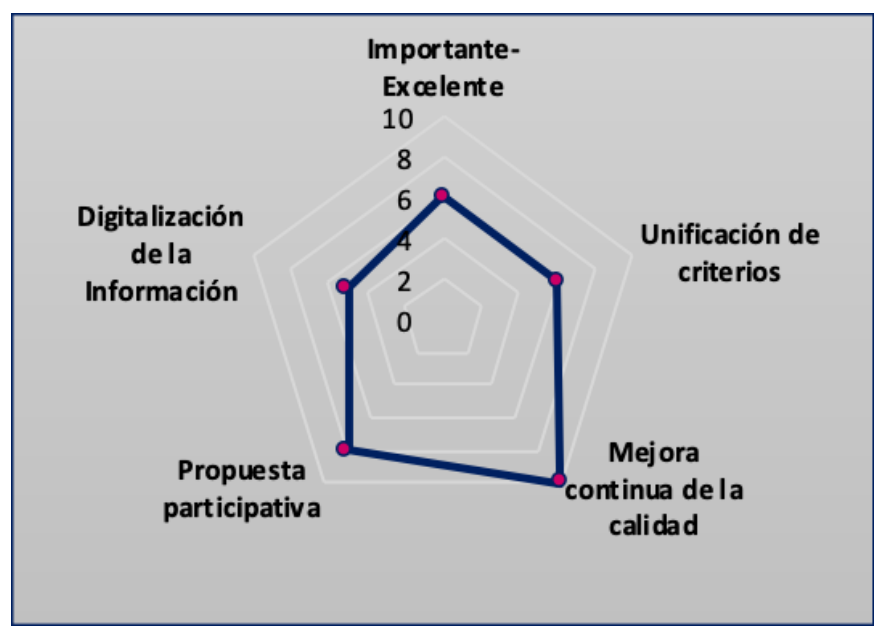

Figura 2. Mejoras identificadas

Seguido tenemos la unificación de criterios y la importancia-excelencia (puntaje 6), donde se considera que uno de los elementos que hacen que el proyecto tenga relevancia es el debate que genera entre diferentes puntos de vista, además de integrar cada opinión y esfuerzo de los participantes en cada indicador y finalizar con su consenso hace que el proyecto se considere interesante e innovador.

Finalmente, el último aspecto considerado, pero no menos importante, es la digitalización de la información (puntaje 5), elemento que es esencial en el proyecto porque se comparte que la información, genera tendencias en la educación superior y crea las herramientas necesarias para el uso de esta.

A más de destacar los tres primeros elementos identificados por los participantes, se puede destacar los siguientes: como importancia en la mejora de la gestión de la Educación Superior, guía para las universidades para gestionar la calidad, y la aplicación de criterios de mejora continua.

\section{B) Mejoras a considerarse en el proyecto de gestión de} la calidad en la Educación Superior

En la Figura 2, se presentan cinco aspectos considerados para mejorar la propuesta, donde el primero es la precisión y priorización de los indicadores (puntaje 10), hace referencia a la definición, fundamentación y análisis que tienen los indicadores, así como mejorar la especificación de las referencias de los mismos, además se enfatiza la unificación de indicadores, es decir la disminución de los mismos o considerar solo los indicadores que sean claves para el desarrollo del proyecto.

El segundo aspecto es la implementación de herramientas tecnológicas para la socialización de la información (puntaje 6). Dichas herramientas son de ayuda para compartir experiencias, comentar o dialogar sobre el proceso del proyecto y contribuir a su mejora constante.

Como tercer aspecto se presenta que los indicadores estén enfocados a los resultados (puntaje 5), es decir que el desarrollo e implementación de los indicadores guíen a la obtención de resultados excelentes ya sean en evaluaciones o para mejorar continuamente la calidad en la educación superior, además de que estén orientados a los diferentes modelos de gestión de cada universidad.

\section{C) Recomendaciones para el proyecto}

En la Figura 3, las recomendaciones principales y con mayor puntaje (10) son: 1) la participación de expertos en indicadores, es decir que para tener una mejor validación del catálogo se debe considerar la presencia de gremios especializados en indicadores, así como la de áreas directivas y aportación de expertos relacionados a cada dimensión de los indicadores; 2) la integración de IES públicas y privadas, hacen que el proyecto tenga una visión más real y se amplié el alcance del proyecto, no solo para instituciones públicas si no para las privadas y que se vaya tomando en cuenta la realidad que tiene cada una.

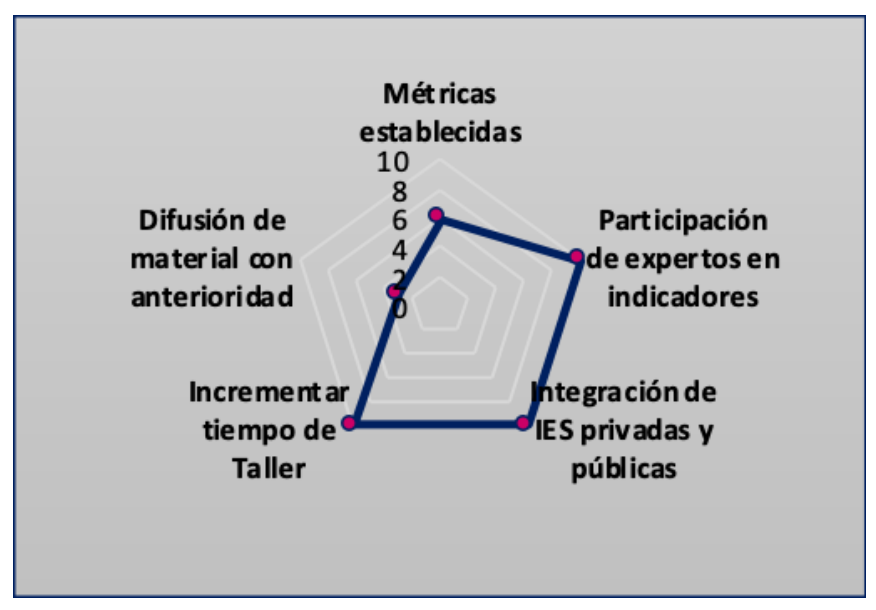

Figura 3. Recomendaciones para el proyecto 
Como tercera recomendación se encuentra el establecimiento de métricas para comparaciones en el análisis de los indicadores (puntaje 6), la misma que es de gran ayuda para determinar cuáles son los fundamentos que se plantea para la consideración de la escala de medición y si la misma puede ser ajustada a diferentes modelos de gestión universitaria.

La última recomendación es que la difusión del material se lo realice con anterioridad (puntaje 3), para tener una mejor concepción sobre el tema u objetivos del proyecto y taller, con el fin de realizar más aportaciones que ayuden en la mejora de este.

\section{DISCUSIÓN}

El modelo generado ¿es el modelo que asegura la calidad de la educación superior?, ¿es otro modelo planteado para las universidades?, ¿con él se solucionan los problemas de calidad en la IES? ¿Promueve un camino hacia una cultura de calidad de las IES?

Se observa que la mayoría de las discusiones se dirigen en ese sentido, a encontrar el modelo ideal que lleve a asegurar la calidad de la educación superior, pero con la óptica puesta en las evaluaciones para la certificación, en discutir al modelo en los rubros que el sistema o que la IES es débil. Para la investigación, la orientación va hacia fomentar una cultura de calidad, hacia convertir al sistema de gestión en una herramienta para la concreción de esa cultura de calidad, para disuadir a las autoridades y los diferentes actores a asumir con responsabilidad sus tareas para asegurar la calidad de las IES y para generar la reflexión en el mismo sistema.

Por otra parte, el modelo propuesto busca aportar a la mejora del oficial propuesto por el CACES (Consejo de Aseguramiento de la Calidad de la Educación Superior) como organismo del Estado obligado a trabajar en la calidad de la educación superior.

Pero como se puede observar en la Figura 1. Valía e importancia del proyecto, los miembros de las universidades indican que este proyecto es importante porque permite a través de un debate e intercambio de experiencias de las mismas universidades integrar sus esfuerzos para lograr mejorar la gestión de la Educación Superior, elementos como Indicadores de calidad generados con una propuesta participativa permitía a las universidades integrar sus experiencias para crear un modelo que se ajuste a sus realidades, esto responde a los estipulado en [16], permite evidenciar la importancia de un conjunto de indicadores que faciliten la evaluación interna orientados a la cultura de calidad.

De la misma manera en la Figura 2. Mejoras identificadas, se visualiza que otro elemento importante es un sistema informático que permita la socialización entre las mismas universidades, con propósito de realizar precisiones y priorizaciones de los indicadores en fusión de la realidad de cada una de las instituciones, respondiendo a la misión y visión de cada una pero que permita mejorar el Sistema completo de Educación Superior.
Al analizar las recomendaciones realizadas de la Figura 3. Recomendaciones para el proyecto, se visualiza la necesidad de un sistema de indicadores que permita realizar comparaciones entre cada una de las universidades con el propósito de evidenciar buenas prácticas en la gestión, así como la calidad es un proceso en el cual deben participar todos los involucrados, pero se da especial importancia a las áreas directivas, expertos en indicadores y gremios especializados en indicadores claves.

Además, se sugiere la inclusión de las universidades privadas ya que son parte del Sistema de Educación Superior con una participación. Por tanto, al revisar los aportes de los participantes y confrontando con la base teórica recopilada se concluye que una cultura de calidad se construye contando con un sistema de medición en este caso son indicadores, pero que se construyan ajustados al contexto universitario, que al mismo tiempo que respeta las particularidades de cada institución permita evaluar las buenas prácticas de gestión de la calidad para ser replicadas. Además, se evidencia la necesidad que este proceso deberá contar con una aplicación informática que permita el intercambio de experiencia y comunicación entre las autoridades universitaria en favor de construir una cultura de calidad con todos los actores universitarios.

\section{CONCLUSIONES}

Al sistema de educación superior y a sus actores se les debe proporcionar la información necesaria de las condiciones de su universidad dentro del contexto nacional, con ello convertirse en actores y practicantes de una cultura de calidad en sus actividades.

La propuesta presentada a discusión, si bien es importante, el verdadero aporte se encuentra en que se convierta en una herramienta para la gestión universitaria, un sistema que sea utilizado por las autoridades universitarias para la planificación, dirección de las IES.

La información clara, exacta y oportuna puesta a disposición las autoridades y actores de las IES les permitirá tomar decisiones más acertadas.

La cultura de calidad requiere un proceso de evaluación, un involucramiento de todos los actores, el mismo debe responder al contexto de cada IES como un proceso de mejora continua.

\section{TRABAJOS FUTUROS}

La propuesta en sí no es el resultado final que se espera de la investigación, por tanto, se seguirá trabajando en lo siguiente:

a) La construcción de una aplicación informática que permita hacerlo utilizable, respetando las particularidades de cada institución pero que a su vez permita intercambiar experiencias.

b) La evaluación de varios proyectos piloto en las universidades del país que permita seguir validando la propuesta y mejorándola.

c) La creación de un modelo de gestión de IES para uso y aplicación de las universidades desde sus experiencias y buenas prácticas acompañado con un proceso de capacitación permanente. 


\section{AGRADECIMIENTO}

El equipo de investigación agradece a la Corporación Ecuatoriana para el Desarrollo de la Investigación y la Academia (CEDIA) por su apoyo y respaldo al trabajo que busca proponer una discusión y un sistema de gestión en procura de lograr institucionalizar la educación superior con calidad.

\section{REFERENCIAS}

[1] J. Ortiz-Herrera, Jorge; Cadena-Vela, Susana; Loza, Edison; Segura, Marco; Perez, Alba; García, "LA EDUCACIÓN SUPERIOR ECUATORIANA : UNA HISTORIA EN BÚSQUEDA DE," LACCEI, no. July, pp. 1-8, 2018.

[2] R. del E. Asamblea Constituyente, "Mandato Constituyente No. 14, , vol. 8 , no. 8 , p. 7,2008

[3] CONEA, "MODELO DE EVALUACIÓN DE DESEMPEÑO INSTITUCIONAL DE LAS Nota," no. 14, pp. 1-92, 2009.

[4] Martínez J, Tobón S, and Romero A., "Problemáticas relacionadas con la acreditación de la calidad de la educación superior en América Latina," Innovación Educ., vol. 17, pp. 79-96, 2016.

[5] E. Ibarra, "Impacto de la evaluación en la educación superior mexicana: Valoración y debates," Rev. la Educ. Super., vol. XXXVIII, no. 149, pp. 173-181, 2009.

[6] E. T. Martínez and M. C. Briceño, "Quality Management Systems as a model of organizational change in the Colombian public sector," Estado del arte del Dir. estratégico, vol. 5, no. 2145-1389, pp. 91-102, 2012.

[7] D. B. Suarez, "Investigación Documental Sobre Calidad De La Educación En Instituciones Educativas Del Contexto Iberoamericano 1 Documentary Research About Quality In Education In Ibroamerican Schools," Entramados Educ. y Soc., vol. 2, no. 2, pp. 107-124, 2015.

[8] G. Hernández, Arcos José Luis, and Sevilla Juan José, "GESTIÓN DE LA CALIDAD BAJO LA NORMA ISO $9001 \mathrm{EN}$ INSTITUCIONES PÚBLICAS DE EDUCACIÓN SUPERIOR DE MÉXICO," 2013.

[9] T. Lopez, "Beneficio de la cultura de calidad en una institución educativa de nivel superior Resumen Primera parte : La calidad Segunda parte : La calidad en la educación superior,” México, 2020

[10] A. Fernández, L. Santini, and J. Ianda, "Una pólemica sobre la calidad de la educación superior," Anuies, vol. 1, pp. 1-7, 2010.

[11] E. Koppel Vintimilla, "Revista de la Universidad de Cuenca / Tomo 57 / Cuenca," Anales, pp. 33-39, 2015.

[12] J. Guzmán, "La calidad de la enseñanza en educación superior ¿Qué es una buena enseñanza en este nivel educativo?," Perfiles Educ., vol. 33, no. Extra 0, pp. 129-141, 2011.

[13] A. del R. M. \& G. Celorio, Hacia una universidad socialmente comprometida. Pais Vasco: HEGOA, 2018.

[14] U. Giraldo, D. Abad, and E. Díaz, "BASES PARA UNA POLÍTICA DE CALIDAD DE LA EDUCACIÓN SUPERIOR EN COLOMBIA."

[15] L. Yzaguirre, "Calidad Educativa e ISO 9001-2000 e n México.," REICE - Rev. Electrónica Iberoam. sobre calidad, Efic. y cambio en Educ., vol. 3, no. 1, pp. 421-431, 2005.

[16] I. Alejandro and S. Durazo, "La Acreditación De La Calidad Educativa Y La Percepción De Su Impacto En La Gestión Académica: El Caso De Una Institución Del Sector No Universitario En México Accreditation of Quality of Education and Its Impact on Academic Management: the Case of a Non-," pp. 305-333, 2013.

[17] J. J. Vesga, "Cultura organizacional y sistemas de gestión de la calidad: una relación clave en la gestión de las instituciones de educación superior," Rev. Guillermo Ockham, vol. 11, no. 2, p. 89, 2013.

[18] R. Hernandez Sampieri, Metodologìa de las Investigaciòn. 2015.

[19] L. Í. Rueda, "Núm. 8.15 de mayo," Investig. y evaluación Cual. bases teóricas y conceptuales, vol. 23, 1999.

[20] M. S.-M. 3 S. Cadena 1, JL García 2, E. Loza-Aguirre 3 , J. Ortiz 1 , A. Pérez 1, "MEDICIÓN DE LA CALIDAD DE LA EDUCACIÓN SUPERIOR.” Palma, España, p. 12, 2018.

[21] M. Armijo, Manuales Planificación estratégica e indicadores de desempeño, CEPAL. Santiago de Chile: Naciones Unidas, 2011. 